\title{
Case Study: Preventing and Resolving Conflict Between Bahamian Nationals and the Haitian Diaspora that Reside in The Bahamas
}

\author{
Abdul D Knowles \\ Director, University of The Bahamas. E-mail: abdulknowles@gmail.com \\ Received: November 26, 2018 \\ Accepted: December 17, 2018 \\ Online Published: December 22, 2018 \\ doi:10.11114/ijlpa.v1i2.3898 \\ URL: https://doi.org/10.11114/ijlpa.v1i2.3898
}

\begin{abstract}
Conflict between Haiti and its Caribbean neighbors often arises because of its large migrant population. In The Bahamas, ethnic, cultural and political reasons have resulted in conflict between Haitians and Bahamians. Bahamians regard Haitians as culturally, ethnically and economically inferior. Haitians are thought to deprive Bahamian nationals of government resources. This has resulted in intolerance and discrimination towards Haitians by Bahamian locals and government bodies. As a result, the basic human rights of Haitians are violated. Stereotypes and mistreatment of Haitians have resulted in anti-Bahamian sentiment and mistrust. Without mediation via international non-governmental organizations (NGOs), the joint-initiatives between the Bahamian and Haitian government will continue to fail. As a result, the ongoing conflict between both groups risks escalating to mass violence.
\end{abstract}

Keywords: Haitian, Bahamian, Migrants, NGOs, Conflict, Initiatives, Mediation

\section{Introduction}

The Bahamas and the Republic of Haiti are close in proximity, yet worlds apart due to the ongoing conflict between Bahamian nationals and the Haitian diaspora that reside in The Bahamas. Since 1804, Haitian migrants have emigrated to The Bahamas in search of better economic opportunities. However, even after The Bahamas, like Haiti, gained independence from a colonial power, the conflict between both parties has continued. Since the conflict began, intolerance has progressed to discrimination and incidences of violence. Both major political parties in The Bahamas, the Progressive Liberal Party (PLP) and the Free National Movement (FNM), and Haitian authorities both in The Bahamas (diplomats) and Haiti (government officials) have developed several joint-initiatives to resolve the issues. Bahamians refer to the issues as the "Haitian problem." Likewise, Haitians regard the issues as the "Bahamian problem." For Bahamians, these issues include; the large migration and assimilation of Haitians into local Bahamian communities. Bahamians believe this results in the increased competition for and the deprivation of limited government resources, mainly healthcare and education. In addition, Bahamians believe the assimilation of the large Haitian population, which includes HaitianBahamians, risk eroding Bahamian culture. Intolerance, mistrust and mistreatment have resulted in Haitians harboring anti-Bahamian sentiments. Haitians accuse the Bahamian Government of abuse and of basic human rights violations. For example, Haitians born in The Bahamas to one Bahamian parent are stateless until the age of 18 when they are permitted to apply for Bahamian citizenship. Without an effective strategy to address this ongoing conflict, mass violence is imminent. Similar treatment of Haitians in the Dominican Republic, also a Caribbean country, have resulted in violence. This qualitative case study, using various conflict theories and models, investigates the ongoing conflict between Haitians and Bahamians by examining the major issues between the parties including previous and current initiatives and strategies used to address the issues. This paper aims to propose effective conflict resolution strategies that could aid both Haitians and Bahamians in addressing the issues before they escalate to violence.

\section{Towards Conflict Between Haitians and Bahamians}

There are many definitions of conflict. However, what best defines the conflict between Haitians and Bahamians in The Bahamas is the perceived divergence of interest and perception by one of the parties that its aspirations are incompatible with those of the other party (Pruitt \& Kim, 2004). Seeds of the conflict between Haitian and Bahamian can be traced back to the colonial occupation of both countries. Haiti's economy continued to spiral due to exploitation by the French after they left in 1804. Coupled with natural disasters, it has become the poorest country in the Western Hemisphere. In 2018, Haiti, with a population of 10.8 million, had a per capita GDP of \$765 USD (Caribbean Community [CARICOM], 2018). With a failing economy, Haitians began migrating to more prosperous neighboring countries, including The 
Bahamas, which in 2018 had a population of 395,361 and a per capita GDP of \$30,352 USD. Haitians are the largest migrant population in the Bahamas, constituting roughly $16 \%$ of the 400,000 population of The Bahamas. The Haitian population is considered the lower economic class of Bahamian society. The gradual influx of Haitians to The Bahamas with cultural stereotypes has resulted in intolerance by Bahamians. Defined as the "Rome Model," intolerance towards Haitians has led to discrimination, which has led to violence (Seton Hall University [SHU], 2015). The "Rome Model" is a good example that explains the progression to violence, however, it does not perfectly explain the likelihood in the Haitian---Bahamian conflict. After "discrimination" and before "violence," struggle (protests) is likely to occur. One Haitian diplomat described Haitians as "peace loving who continue to thrive during adversity" (Mesamours, 2018). As citizens of the world's poorest nations, Haitians continue strive amidst discrimination by both its Caribbean neighbors and the international community. Only after years of protests is violence likely. Mistreatment and abuse by Bahamians have resulted in mistrust and anti-Bahamian sentiments by Haitians. The Bahamas, which is in close proximity to the United States is influenced by American culture and politics. The mistreatment of Haitians by Bahamians is fueled by the United States' characterization of Haitians and Haiti as "high poverty and unemployment, low educational achievement and poor health conditions" (Refworld, 2012). While campaigning in 2016, the United States President, Donald Trump allegedly called Haiti a "shithole" country. (Daugherty, 2018). The belief by Bahamians and Americans that all Haitians are poor economic migrants is faulty thinking. Although Haitian authorities acknowledge there is a Haitian migrant problem, many are refugees stemming from Haiti's turbulent political past. In the 1980s and 1990s, many Haitians refugees were fleeing a brutal dictatorship. Cultural misunderstandings between Haitians and Bahamians have fueled the conflict. This is discussed below.

\section{Cultural and Ethnic Causes of the Haitian-Bahamian Conflict}

During times of conflict, certain psychological states, which include negative views and attitudes toward the other group, dehumanization, de-evaluation, hostility and competitiveness, can develop between groups (Nan, Mampilly \& Bartoli, 2011). The source of conflict between Bahamians and Haitians are diverse. In addition to historical circumstances, differences in language and culture play a role in the conflict. Haitians and $85 \%$ of Bahamians are both descendants of West-African slaves. Yet, Bahamians are racist towards Haitians. According to a Bahamian university student, "Haitians are easily distinguished from Bahamians due to racial features. Haitians tend to be darker complexion." Haitians are chastised for speaking Creole, which Bahamians consider "broken-French." Although most Haitians and Bahamians share Christian values, Haitians are also stereotyped as practicing voodoo (an African-based religious practice), portrayed in the West, including The Bahamas, as a dark and evil cult of devil worship, dominated by black magic, sacrifice, and pinstuck voodoo dolls (Guynup, 2004). Over decades, these negative stereotypes of Haitians have permeated every aspect of Bahamian society. In The Bahamas, the Haitians are proud because their community and culture which are considered more unified than Bahamian culture (Editor, 2018). One Bahamian subject interviewed summed up the general thinking of Bahamians: "most Haitian-Bahamians (persons born in The Bahamas with both Haitian and Bahamian parents) living in The Bahamas, chose to identify with their Haitian heritage. Therefore, they are not loyal Bahamians and not entitled to Bahamian citizenship" (Clarke, 2018).

In sum, Bahamians and Haitians each consider themselves culturally superior. This cultural divide plays a role in the ongoing conflict. To resolve the conflict, both parties have specific aspirations, defined as "mental representations of things that each Party strives for or believes it must achieve" (Pruitt \& Kim, 2004). The aspirations of both parties are discussed below.

\section{Aspirations of Haitians in its conflict with Bahamians}

First, the major tension between Haitians and Bahamians stems from the implementation of Bahamian government laws and policies primarily aimed at Haitians. One such policy is the non-recognition of Haitian-Bahamians born in The Bahamas to both Haitian and Bahamian parents. In 2014, Bahamian authorities implemented a new immigration policy requiring everyone living in the Bahamas to have a passport of their nationality. This replaced certificates of identity that were previously issued, including persons born to foreign parents legally residing in The Bahamas (Turnquest, 2014). In addition, identification documents previously issued to children of illegal Haitian residents were discontinued (Wells, 2014). This leaves many Haitian-Bahamian stateless. After the age of 18, Haitian-Bahamians can apply to be legally recognized, however, approval is not guaranteed. Many Haitian-Bahamians hold Haitian passports after turning 18. However, according to one Haitian diplomat interviewed, "the Bahamian government has created a maze of rules and processes for Haitian-Bahamians seeking citizenship. It can take between 2-6 years to obtain citizenship, which is not guaranteed" (Mesamours, 2018). Therefore, securing legal documentation which recognizes them as legal residents is a basic goal of Haitians, since without documentation, they are unable to accomplish things necessary for their livelihoods. These include, opening a bank account, getting a driver's license, buying a home, etc. Previously, children of Haitian migrants were permitted to attend government school with Haitian identity cards. However, without Bahamian residency, it has become increasingly difficult. The immigration status of Haitian immigrants has made them vulnerable 
to human rights abuses, including discrimination in access to education and health services. Second, Haitians aspire for raids on their communities by Bahamian immigration to end. According to a Haitian diplomat, "Haitians taken during these raids on their communities are often victimized and denied basic human rights. They are denied due process in asylum applications and deportation proceedings" (Mesamours, 2018). Third, Haitians would like a clearer and efficient process in securing work permits. As one Haitian diplomat points out, "Haitians play an essential role in Bahamian society, because they do jobs that many Bahamians refuse to do, such as working in sanitation services, and yard work, etc." (Mesamours, 2018). However, the Bahamian government maintains its unfair immigration policies, and the immigration process for Haitians is often much lengthier compared with other immigrants.

\section{Aspirations of Bahamians in Its Conflict with Haitians}

The aspirations of the Bahamian authorities are as follows. First, the primary goal of the Bahamian government is to stop the flow of Haitian migrants to The Bahamas. Thus, it seeks assistance from Haitian authorities in stemming the tide. Second, The Bahamian authorities believe the burden of the citizenship issue for Haitians born in The Bahamas rests with Haiti. In recent reports, The Bahamas Immigration Minister stated: "If you're born in The Bahamas to non-Bahamian parents you have a right to apply (for citizenship) at the age of 18. You are not Bahamian until then, end of, full stop. The lack of clarity over the entitlement to Haitian citizenship for second and third generation children born in this country is an issue for the Haitian government, not The Bahamas. Therefore, these persons are not "stateless", as Haitians and international NGOs claim." (Turnquest, 2018). The pursuit of limited resources often results in conflict (Pruitt \& Kim, 2004). Bahamians believe Haitians exploit the governments' limited resources and don't contribute to society. The large number of Haitian enrolment in government schools, which increases annually, results in overcapacity. For example, in a 2005 survey, Haitians accounted for 10.67\% of the 39, 742 students enrolled in Public schools (See table 1).

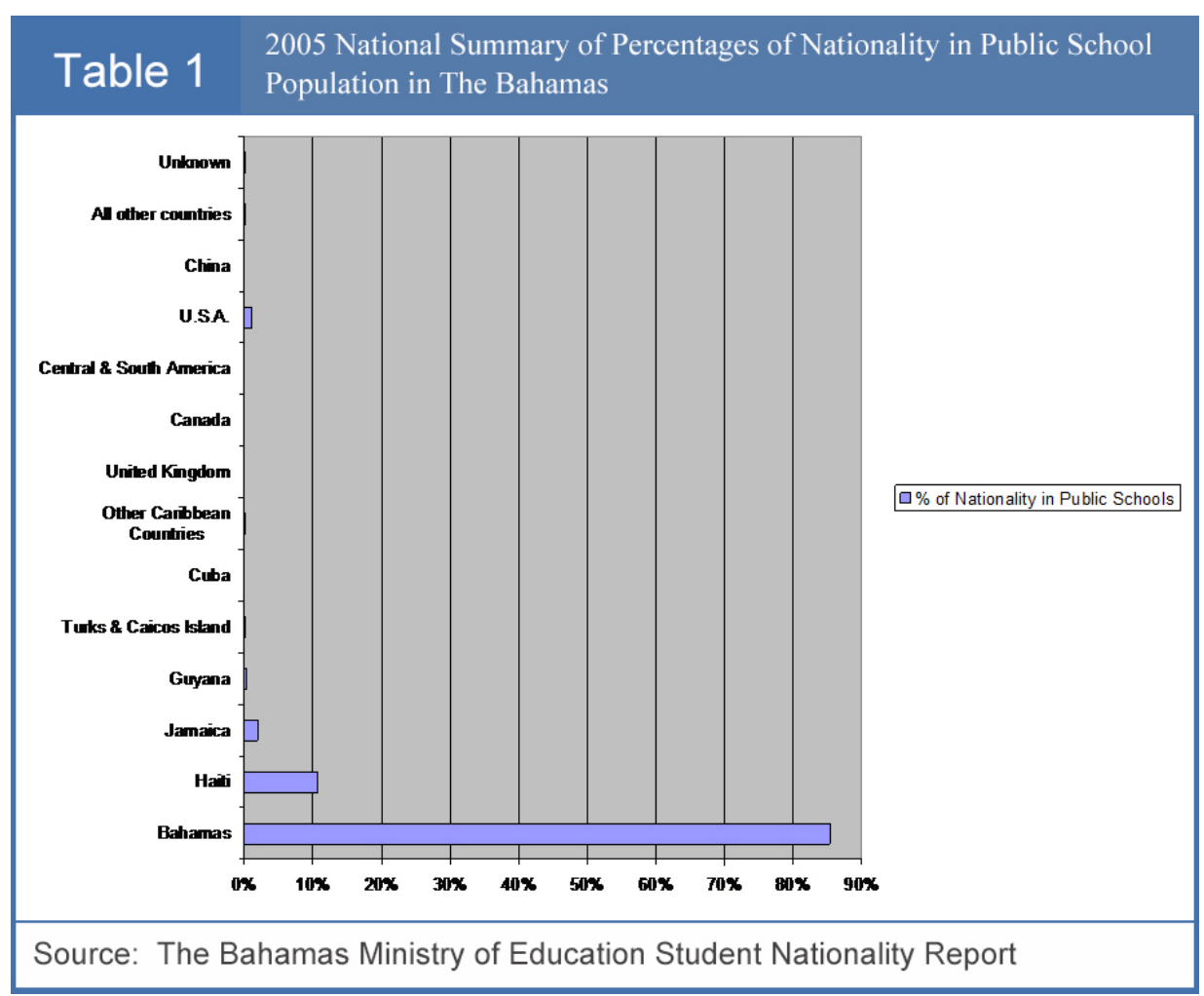

Haitians accounted for $72.81 \%$ of the 5826 non-Bahamian students enrolled in public schools (See table 2). Therefore, Bahamian children lose opportunities to attend key government schools. Bahamians also have "zero-sum thinking," believing that the other's gain is one's loss (Pruitt \& Kim, 2004). Exacerbating the issue is the belief that Haitian migrant children spread of diseases such as scabies in primary schools (Wells, 2015). 


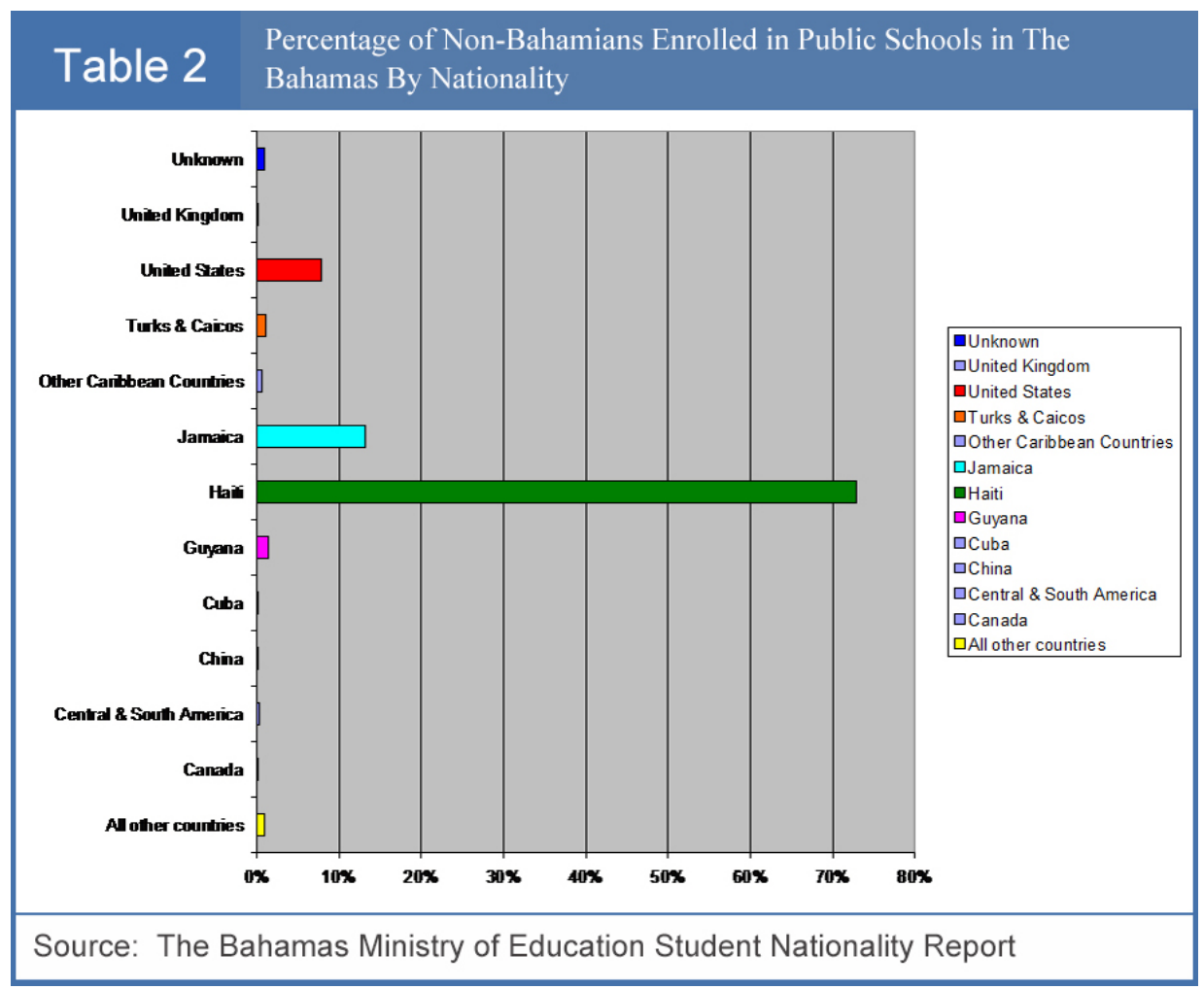

This faulty thinking results in Bahamians thinking they have to compete for government health services and will lose opportunities to see practitioners in a timely manner due to a large number of Haitians seeking similar services. Although necessary, identity cards are not a mandatory requirement for healthcare at government hospital and clinics. Another problematic area for Bahamians is cultural integration. The fertility rate of Haitians is higher than that of Bahamians. Thus, Bahamians argue their identity is being threatened as a result of Haitian integration into Bahamian society. According to one Bahamian university student, "the large number of Haitians, and off spring from intermarriages between Bahamians and Haitians threatens the Bahamian gene-pool" (Clarke, 2018). Bahamians accuse Haitians who are legally employed to work in The Bahamas of taking Bahamian jobs. In addition, Haitians are accused of contributing little to the local economy. Bahamians think all Haitian earnings are wired back to Haiti. Bahamians believe Bahamian authorities should limit the number of work permits to Haitians. However, according to a chief Bahamian immigration Minister, Bahamians are unwilling to do the type of jobs Haitians are willing to do.

\section{Previous and Current Initiatives for Addressing the Conflict}

Over the years, both parties have established initiatives to help de-escalate the conflict. The Bahamas and Haiti agreed to undertake joint initiatives that could contribute to reducing the flow of migration to The Bahamas and resolve the identification and citizenship issue of Haitian-Bahamians (Cartwright-Carrol, 2018). These initiatives entail the Haitian government establishing a center at its Embassy in The Bahamas for the collection of identification documents for Haitian citizens. This would provide documents for Haitian-Bahamians that are denied citizenship and remain "stateless" until the age of 18. However, this initiative which mostly protects Bahamian interests will fail because Haitian-Bahamians seek Bahamian identity documents to receive Government benefits. According to one Haitian-Bahamian university student, university education is cheaper for Bahamian nationals. Without Bahamian citizenship, Haitian-Bahamians pay out-ofstate tuition, which is higher (Duchley, 2018). In March 2013, work permit regulations were made more restrictive by a "Bahamian First" initiative. This resulted in the Bahamian government tightening its migration policies, leading to an increase in detentions and deportations (Intelligence Unit, 2015). This initiative remains controversial both domestically and internationally because it makes it difficult for Haitian migrants to receive work permits, thus threatening their livelihoods. "It is also controversial with both Haitian diplomats and international NGOs. According to one diplomat, Haitians are often mishandled, abused and denied due process during detentions by Bahamian officials (Mesamours, 2018). In 2017, Amnesty International condemned the widespread ill-treatment of Haitian migrants (Amnesty, 2017). The Bahamian NGO, "RIGHTS Bahamas" have threatened lawsuits against Bahamian authorities before international human rights bodies due to widespread abuses of Haitian migrants by the Bahamas Immigration Department (Turnquest, 2018). According to a Bahamian NGO President, Bahamian authorities are "hunting people born in the Bahamas of Haitian descent and expelling them illegally back to Haiti" (Turnquest, 2018). Since coming to power in 2017, Bahamian 
government leadership has promised more dialogue to established initiatives with Haitian government officials in The Bahamas. In addition, the Bahamian government planned to work collaboratively with Haitian officials. This entails, trips to Haiti to discuss with Haitian officials the migration challenges as well as establish joint economic initiatives (Turnquest, 2017). The Prime Minister of The Bahamas indicated the Bahamian government has set up a large task force, which includes representatives of the Haitian community, to help better assimilate Haitian residents from shantytowns it plans to remove. He envisions Haitian Bahamian communities (Tribune, 2018). However, as previously shown, these joint initiatives do not resolve the issues. Establishing Haitian-Bahamian communities does not address the root causes of the conflict, stemming from cultural stereotypes. It also does not resolve the mistrust Haitians and Bahamians have towards each other. The Bahamian government needs to established joint-initiatives with NGOs like the "League for Haitian Pastors" (LHP)" and "Bahamas Islands Baptist Association" (BIBA). Leaders of both NGOs have influence within both Haitian and Bahamian communities. In addition, NGOs appear to take a harder stance against the Bahamian authorities than Haitian officials. For example, LHP, a faith-based group has urged Bahamian authorities to negotiate an agreement to allow Haitian residents to purchase and rebuild 11 Haitian communities ("shantytowns") that are often the target of government raids and demolitions (Scavella, 2014). NGOs like "BIBA" have taken a softer approach in trying to resolve the conflict between Haitians and Bahamians by mainly encouraging dialogue between Haitian leaders and Bahamian authorities (Scavella, 2014). However, international NGOs with louder voices, like "Amnesty International" and "Human Rights Watch," play an important role in resolving the conflict and bringing about meaningful change.

\section{Strengths and Weaknesses of Initiatives (Continued)}

As previously mentioned, the Bahamian and Haitian authorities have held dialogues agreeing to joint initiatives. In theory, these joint-initiatives are good, but in practice they appear to mostly protect Bahamian interests. The migration of persons in search of better economic standards is a global phenomenon. Given Haiti's economic conditions, Haitians will continue to pursue better economic conditions. Bahamian authorities, with the aid of the UNHCR, need to establish an immigration policy that is friendlier to Haitians. As one Haitian diplomat stated, Haitians are willing to follow the rules and apply for the necessary work permits, but Bahamian government makes it extremely difficult and detains Haitians without questions (Mesamours, 2018). Without third-party involvement, there will continue to be oversight on issues with Haitians coming up short at the table. Although there is high unemployment in The Bahamas, there is a shortage of low-skilled laborers in The Bahamas to perform duties most Bahamians shun. Establishing an immigration initiative for these jobs would encourage Haitians to apply at respective Bahamian embassies for these work opportunities and travel legally to The Bahamas, and reduce the number of illegal migrants in The Bahamas. This initiative would also help rebuild trust between Haitian and Bahamians within The Bahamas. Bahamians would help eliminate the stereotypes and suspicions that all Haitian migrants in The Bahamas are illegal. However, due to the large Haitian population, Bahamian authorities should implement a quota for these coveted positions. Because it is evitable that most Haitian-Bahamians will apply for Bahamian citizenship after the age of 18 , Bahamian authorities should grant automatic citizenship to Haitians with one Bahamian parent. Bahamians often complain that Haitian-Bahamians identify with their Haitian heritage. This is likely the result of Bahamian authorities' delayed recognition of their Bahamian heritage. Granting automatic citizenship would not only help Haitians feel more Bahamian and become productive Bahamian role models, it would also help improve the international image of The Bahamas. Some of the most successful grassroots entrepreneurs in The Bahamas are of Haitian descent. Haitians would play a crucial role in helping Bahamian authorities achieve its "Vision 2040" initiative-a comprehensive policy framework intended for national development, decision making and investment. The BahamasHaiti joint initiative to tackle the Haitian migrant issue will fail because Bahamian authorities are not genuine in its pursuit to finding a mutually beneficial solution. Currently, international rights groups consider The Bahamas a human rights violator and a contributor to the global population of stateless persons. Bahamian authorities are not troubled by such accusations. This is evident from the Head of the Bahamas Department of Immigration, who stated "immigration officers have no intention of changing the way it apprehend illegal immigrants and will be stepping up efforts despite criticism from human rights groups" (Dorsett, 2017). Thus, Haitian authorities have continually proposed negotiation as a key strategy to resolving its conflict with Bahamian authorities (Turnquest, 2014). To ensure fair and successful joint initiatives, Haitian authorities prefer international NGOs like the "International Organization for Migration" and the "UN High Commissioner for Refugees" be part of its discussions with Bahamian authorities. Global rights organizations such as Amnesty International support the Haitian position, and often express concern about The Bahamas immigration policy, stating it has resulted in human rights violations. In addition, Amnesty International argue the "Haitian-Bahamian community have been disproportionately targeted by Bahamian immigration officials" (Wells, 2014). However, Bahamian authorities shunned meetings with third-parties thus rejecting multi-lateral negotiation. Bahamian authorities question the credibility of NGOs, believing that international NGOs like "Amnesty International" misunderstand the Haitian-Bahamian conflict, and that their concerns and claims are "dead wrong" and "badly mistaken" (Virgil, 2014). The position of The Bahamian authorities position is that, because of the criminality involved in immigration, these new immigration rules are necessary (Wells, 2014). 


\section{Strategies for Peace}

There is a need for Bahamian authorities to investigate what lies beneath the ongoing conflict between Haitians and Bahamians. Because intolerance and discrimination are underlying causes of the conflict, Bahamian authorities must take a holistic approach to address the conflict. Peacemaking begins first as an idea, an aspiration, or hope for harmony (Nan, Mampilly \& Bartoli, 2011). Although the conflict between Haitians and Bahamians has yet to escalate to mass violence, it doesn't mean it is unlikely to happen. According to one expert, if there is no change in the relational system, hostility accumulates incrementally until a catastrophic event occurs (Wood, 2018). Although rare, there are reported incidences of violence between both groups. Some include physical abuses by members of Bahamian law enforcement. In addition, there are incidences of school-yard fights between Haitian and Bahamians, often the result of bullying. However, the conflict has not continued to escalate because of a perceived stalemate. Both parties perceive it cannot make further progress in the conflict at an acceptable cost or risk (Pruitt \& Kim, 2004). This is evident from numerous dialogues between Haitian and Bahamian authorities. However, dialogue alone will not resolve the conflict. A Haitian diplomat has proposed a "creative social dialogue" (Mesamours, 2018). However, as previously stating, years of dialogue have not produced the desired outcome for both parties. Haitian and Bahamian authorities should engage in negotiation or some form of Alternate Dispute Resolution (ADR) such as mediation, which according to scholars, ordinarily grow out of perceived stalemate (Pruitt \& Kim, 2004). Negotiation should not be in original forms, that is, parties using contentious tactics to win or a bi-lateral effort in which both parties work side by side to solve the problem jointly (Pruitt \& Kim, 2004). Negotiation should be done via a neutral third-party. Therefore, mediation in which a mediator helps facilitate dialogue in a structured multi-stage process to help parties reach a conclusive and mutually satisfactory agreement (Wood, 2018), is the best option. Marie Dugan's conflict theory is a good perspective for evaluating and proposing effective solutions for the conflict between Haitians and Bahamians. In her article "A Nested Theory of Conflict," Maire Dugan stated, mediating the specific issue might not effectively address a conflict (Dugan, 1996). Thus, Dugan proposed four levels in which issues that exist in a "nest" of systems, can be analyzed (Dugan, 1996). First, the "issue-specific" level, which in the Haitian-Bahamian conflict would address the migrant and immigration issues, but alone would not resolve the conflict, since they are not the root causes. Racial prejudice and discrimination are some reasons behind discriminatory immigration policies. Therefore, Bahamian and Haitian authorities must address the "relational" issues, which stems from problems having to do with the interaction patterns of the parties and their feelings towards each other (Dugan, 1996). These include the negative stereotypes, racial prejudice, discrimination and mistrust both Haitians and Bahamians have towards each other. For example, according to one Haitian university student who attended secondary government schools in The Bahamas, although both the majority of Bahamians and Haitians are of a similar race, there is racial prejudice. Haitian students are often bullied by Bahamian classmates (Richards, 2018). Lastly, the "structural system level" which examines the broader relationship between Haitians and Bahamians, must be addressed. Most of the issues regarding discrimination in employment, education, and immigration and racial sentiments are widely held and known throughout Bahamian and Haitian society. These issues are exacerbated by media accounts (local newspapers and television broadcasts) of current events which shape attitudes of Bahamians. Since few Haitians in The Bahamas read Bahamian newspapers or have access to television, their exposure is mainly via evening Creole radio programs. These weekend programs also shape Haitian attitudes towards Bahamians. Dugan considers the "structural: subsystem issues," which stems from inequities built into the social system, most effective and practical for addressing conflicts (Dugan, 1996). In this level, Bahamian and Haitian authorities could initiate change at schools (including universities) and churches, etc. by working with educators, pastors and other community leaders come to develop a structural plan to change relationships and how Bahamians and Haitians interact.

\section{Why Mediation?}

Peacekeeping can help manage conflict. However, by itself it cannot settle disputes. Negotiation and mediation, common methods for resolving disputes, preserve independent decision-making. Both parties can accept or reject suggestions made by the outsiders. Bahamian authorities are unlikely to accept arbitration because of its binding nature and the general attitude that The Bahamas doesn't need outside interference. In a recent interview with a local paper, the Bahamian Prime Minister, Hubert Minnis, rejected the need for the IMF to interfere in Bahamian fiscal matters the way it did in Haiti. He stated: "We have our own fiscal affairs to deal with, and from that perspective, we don't need to look at others, we simply need to say, 'this is our reality, and this is why we are making decisions" (Bowe, 2018). Also, in a call for a change of policy in order to provide greater rights to Haitians in The Bahamas, the Head of the Department of Immigration responded: "Bahamian laws are sovereign laws, and I respectfully suggest that persons should not dictate or try to dictate to us what our laws should be" (Turnquest, 2018).

To address the ongoing conflict, Bahamian and Haitian authorities often engage in what scholars define as "Track One Diplomacy" (government-to-government) diplomacy (Nan, Mampilly \& Bartoli, 2011). However, this type of diplomacy has yet to yield a positive result. It also would not address the "relational issues" as previously mentioned. Both parties 
should start with smaller goals that build mutual understanding. "Track Two Diplomacy" which is non-governmental in nature, innovative and flexible (Nan, Mampilly \& Bartoli 2011), "Track Four Diplomacy" (citizen exchanges), and "Track Five Diplomacy" (Nan, Mampilly \& Bartoli, 2011) such as establishing a joint student organization at a university, or organizing academic exchanges between Haitian Universities the University of the Bahamas, are positive options. Institutions like the University of The Bahamas (UB), the premier institution of higher learning in The Bahamas should play an important role in promoting educational and cultural exchanges between groups. Establishing a Haiti-Bahamas student association (student organization) at UB would help better facilitate inter-cultural dialogue which would help remove barriers and help resolve the conflict. According to a Haitian diplomat, the Haitian Embassy would welcome and support such student association. As an educational community that encourages student social groups, University of The Bahamas administrators are likely to support this initiative as well.

Haiti and The Bahamas are both the Permanent Member States of the Caribbean Community (CARICOM) and Organization of American States (OAS). OAS, which was established in order to achieve among its member states, "an order of peace and justice, to promote their solidarity and strengthen collaboration" (OAS Charter, Article 1). CARICOM is called to prioritize growing migration issues (CARICOM, 2018). According to Chief Haitian diplomat, OAS and CARICOM could act as powerful mediators (Mesamours, Although Bahamian authorities shun third-party involvement, it would likely consider third-party intervention by regional organizations, as proposed by Haiti. However, such thirdparty involvement in the conflict should be considered carefully since some of its Member States, also dealing with the "Haitian problem" is likely to side with Member States with similar issues. Therefore, international NGOs should serve as third-parties.

\section{Towards Long-Lasting Peace}

Recently, the Bahamian Government leadership acknowledged the need to address "misunderstandings" in the government dealing with undocumented Haitian migrants in The Bahamas (Tribune, 2018). Haitians, on the other hand, needs to build trust with Bahamians. According to Counselor Mesamours, the intolerance and discrimination Bahamians have towards Haitians, and the animosity Haitians have towards Bahamians is unlikely to escalate into violence. He advocates dialogue as the way forward. The dialogue between Haitian and Bahamian officials, including that between Bahamian officials and the leaders of the "League of Haitian Pastors." However, dialogue alone will not resolve the conflict between the demands of both parties at large. Discussions and negotiations will not end the demand from Bahamian authorities to restrict the flow of Haitian economic migrants to The Bahamas. Nor, will Haitian authorities give up their needs for better human rights for Haitian descendants in The Bahamas. With the assistance of a third party, and set goals, peace is possible. However, as scholars point out, the third party must define more realistic short-term and intermediate goals (Nan, Mampilly \& Bartoli, 2011). First, Bahamians needs to address the root causes by establishing a policy to combat the long-standing discrimination against those of Haitian descent. As previously mentioned, educational institutions, including public schools with a large Haitian migrant population, are great places to start.

\section{Conclusion}

There has undeniably been a history of conflict between the Haitian and Bahamian population in The Bahamas. As the largest migrant population in The Bahamas, Haitians play an important role in The Bahamas. Previously, Bahamian authorities have not been genuine in their attempts to resolve the Haitian-Bahamian conflict. Instead, to win elections, Bahamian politicians whip up xenophobic sentiments as a distraction from domestic problems. Bahamian authorities need to work towards genuinely resolving the conflict with their Haitian counterparts. Neglecting to resolve this conflict will continue to have negative consequences for the Bahamas, both domestically and internationally. Resolving this conflict includes establishing initiatives that are beneficial and acceptable to both parties. To ensure this occurs, mediation with third-party NGOs is necessary. Haitians have often been the losing party in these joint initiatives, which are for the benefit of Bahamian interests. With a growing Haitian population, some believe a continuation of discriminatory practices by Bahamian authorities would lead to an exodus of Haitians from The Bahamas. While this is possible with Haitian migrants, an increasingly vocal Haitian-Bahamian group are likely to continue the struggle (protest) for the equal rights they deserve. Thus, intolerance and discriminatory practices will lead to protests then violence. It is only through mediation via international NGOs, that The Bahamas, a nation "steeped in a culture of discrimination and human rights abuses," can improve its international image and live up to its slogan, "It's Better in The Bahamas."

\section{References}

Amnesty (2017), Amnesty Int'l Critical of Jamaica, Haiti, The Bahamas, Jamaica Observer, February 27, 2017, http://www.jamaicaobserver.com/news/Amnesty-Int-1-critical-of-Jamaica--Haiti--The-Bahamas

Bowe, G. (2018, July 18) Bowe Critical of PM's Haiti-Bahamas Comparison, The Nassau Guardian

Cartwright-Carroll, T. (2018a, February 26), PM in migrant talks in Haiti, The Nassau Guardian 
Cartwright-Carroll, T. (2018b, July 31) The Nassau Guardian, https://thenassauguardian.com/2018/07/31/haitian-pastorwants-shanty-residents-up-first-for-land-purchases/

Daugherty, A (2018, September 11), Trump called Haiti a 'sh-hole' campaigning in Miami in 2016, Woodward's book says, Miami Herald, https://www.miamiherald.com/news/politics-government/article218202540.html

Dorsett, S. (2017, December 28), Haitian Illegals - 114 To Fly Home, Tribune 242, http://www.tribune242.com/news/2017/dec/29/haitian-illegals-114-fly-home/

Intelligence Unit (2015, February 27), Government Urged to Improve Detention Conditions, The Economist. https://country.eiu.com/article.aspx?articleid=1942925378\&Country=Bahamas\&topic=Politics\&subtopic=Forecast \&subsubtopic $=$ Political + stability\&u $=1 \&$ pid $=777248861 \&$ oid $=777248861 \&$ uid $=1$

Guynup, S. (2004, July 7). Haiti: Possessed by Voodoo, National Geographic. https://news.nationalgeographic.com/news/2004/07/haiti-ancient-traditions-voodoo/

Dugan, M. (1996). A Nested Theory of Conflict. A Leadership Journal: Women in Leadership- Sharing a Vision: 1.1. pp. 9-20

Pruitt, D.G., \& Kim, S.H. (2004). Social Conflict: Escalation, Stalemate, and Settlement (3 ${ }^{\text {rd }}$ ed.). McGraw Hill

Joseph, F. (2014), The Treatment of Haitian Bahamians in Bahamian Society, International Journal of Bahamian Studies, Vol 20, No. 1. http://journals.sfu.ca/cob/index.php/files/article/view/226/266

Nan, S.A., Mampilly, Z. C., \& Bartoli, A. (ed), (2011). Peacemaking: From Practice to Theory. Praeger Security International, Santa Barbara, CA

Mesamours, H. (2018). Haitian-Bahamian Conflict (Interview)

Richards, D. (2018, November 14) (Interview)

Scavella, N. (2014, November 15). Pastor Voices Concern Over Immigration Raids, Tribune242, November 15, 2014, http://www.tribune242.com/news/2014/nov/05/pastor-voices-concern-over-immigration-raids/

SHU (2015, April 25). Religious Intolerance \& Discrimination in the West [PowerPoint slides]. Retrieved from https://www.youtube.com/watch?v=XXI9cq-68FM\&feature=youtu.be

Turnquest, A. (2014, November 14). Haiti Delegation to Have Talks, Tribune 242. http://www.tribune242.com/news/2014/nov/17/haiti-delegation-have-talks/

Turnquest, A. (2017, November 21). US Action On Haitians Will Affect Bahamas, Tribune 242. http://www.tribune242.com/news/2017/nov/22/us-action-haitians-will-affect-bahamas/

Turnquest, A. (2018a, February 16). Rights Bahamas To File Suit Against Govt Before International Bodies, Tribune242. http://www.tribune242.com/news/2018/feb/16/rights-bahamas-file-international-suit-against-gov/

Turnquest, A. (2018b, October 15). Haiti Must Deal with Children of Migrants, Not Bahamas, Tribune 242. http://www.tribune242.com/news/2018/oct/15/haiti-must-deal-with-children-of-migrants-not/

Virgil, K. (2014, November 14). Mitchell in Talks in US to Clarify Immigration 'Misconceptions. Tribune 242. http://www.tribune242.com/news/2014/nov/19/mitchell-talks-us-clarify-immigration-misconceptio/

Wells, R. (2015, February 10). Public is Crucial to Preventing The Spread of Scabies, Tribune 242. http://www.tribune242.com/news/2015/feb/10/public-crucial-preventing-spread-scabies/

Wells, R. (2014, November 18). Human Rights Violations Concern From Amnesty. Tribune 242. http://www.tribune242.com/news/2014/nov/18/human-rights-violations-concern-amnesty/

Wood, D. (2018), Peacemaking and Peacekeeping [PowerPoint slides]. Retrieved from

Tribune (2018, May 25). PM Calls For Love Between Bahamians And Haitians. http://www.tribune242.com/news/2018/may/25/pm-calls-love-between-bahamians-and-haitians/

MOE, Ministry of Education, Science and Technology, The Planning and Research Section, The Ministry of Education Student Nationality Report, 2005

MOE (Ministry of Education) (2016). Science and Technology, The Planning and Research Section, 2015-2016 Student Nationality Report, 2016.

CARICOM (2018, November 21). Caribbean Community. Retrieved November 21, 2018 from https://countryeconomy.com/countries/groups/caribbean-community

CARICOM, Communique: Twenty-First Meeting of the Council for Foreign and Community Relations (COFOR), Nassau, 
The Bahamas, 7-8 May, 2019, CARICOM, May 9, 2018, https://caricom.org/mediacenter/communications/communiques/communique-twenty-first-meeting-of-the-council-for-foreign-andcommunity-relations-cofcor-nassau-the-bahamas-7-8-may-2018

Editor (2014, December 14). Haitian Activists Speaking Out On The Bahamas. Tribune 242. http://www.tribune242.com/news/2014/dec/18/haitian-activists-speaking-out-on-the-bahamas/

OAS Organization of American States (2018, November 20). Retrieved November 20, 2018 from www.oas.org/en/member_states/default.asp

\section{Copyrights}

Copyright for this article is retained by the author(s), with first publication rights granted to the journal.

This is an open-access article distributed under the terms and conditions of the Creative Commons Attribution License which permits unrestricted use, distribution, and reproduction in any medium, provided the original work is properly cited. 